\title{
Determination of Tip Shape in Fungal Hyphae
}

\author{
P. T. SAUNDERS \\ Department of Mathematics, Queen Elizabeth College, Campden Hill Road, \\ London $W 87 \mathrm{AH}$ \\ AND A. P. J. TR INCI \\ Department of Microbiology, Queen Elizabeth College
}

(Received 31 August 1978)

INTRODUCTION

Extension of a fungal hypha is the result of primary wall growth at its tip (Trinci, 1978); the apical region involved in this growth is called the extension zone. Precursors required for primary wall growth are probably synthesized in a relatively long region of hypha (the peripheral growth zone) distal to the tip and then transported to the extension zone where they are incorporated into the tip wall (Trinci, 1978); some wall precursors are transported in vesicles which fuse with the tip membrane, liberating their contents into the extending wall. The wall of the extension zone has a constant thickness (usually about $50 \mathrm{~nm}$ for mycelial hyphae) and is made up of a network of apparently randomly orientated microfibrils (diameter of about $10 \mathrm{~nm}$ ) embedded in an amorphous matrix. The length and shape of the extension zone of a hypha growing at a linear rate remain constant with time. Since the volume of the extension wall remains constant it follows that the rate of addition of new wall material must equal the rate at which wall at the base of the extension zone becomes 'rigidified', i.e. no longer expands in surface area (Robertson, 1965).

The results of Robertson (1958) and Robertson \& Rizvi (1968) suggest that wall elasticity is maintained for a longer period at the tip than in distal regions of the extension zone. Robertson (1958) found that when extension growth of a hypha of Fusarium oxysporum was stopped by an osmotic shock, the entire extension zone became rigidified within about $40 \mathrm{~s}$; loss of wall elasticity started at the base of the extension zone and spread rapidly towards its tip. Regrowth from such 'rigidified' tips took the form of sub-apical branches, i.e. the tip wall was softened locally to form branch initials. However, Robertson found that if re-growth of osmotically shocked hyphae started before the extension zone had become completely rigidified, then extension occurred from the residual elastic wall at the extreme tip.

Steele \& Trinci (1975) defined the extension zone expansion time $\left(Z_{t}\right)$ of a hypha as the period required for a hyphal tip to expand from its minimum to its maximum diameter. They suggested that $Z_{t}$ gives an estimate of the period which elapses between insertion of elastic wall material at the tip of a hypha and its rigidification at the base of the extension zone. From Robertson's (1958) data we estimate that a hypha of Fusarium oxysporum has a $Z_{\mathrm{t}}$ of about $95 \mathrm{~s}$; this estimate may be compared with the $40 \mathrm{~s}$ which Robertson (1958) observed was required to complete rigidification of the tip wall (see above).

In this paper we suggest that wall elasticity is the chief factor which determines the shape of the tip, and we derive a differential equation which provides a relation between the rigidity of the wall and its curvature. This is in principle sufficient to allow us to make predictions of tip shape based on hypotheses about rigidity, but because the equation is difficult to integrate it is more likely to be used to estimate the rigidity, which is hard to measure directly, from measurements of tip shape. 


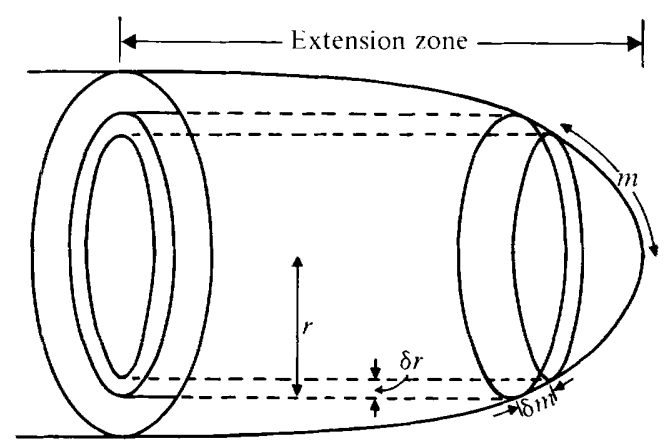

Fig. 1. Idealized model of a hyphal tip illustrating that the growth of a ring of wall of width $\delta m$ depends on wall material supplied through an annular ring of width $\delta r$. The diagram shows an oblique view of the tip.

Most previous authors appear to have supposed that the shape of the tip is determined by variations in the rate of supply of wall precursors to different parts of the tip wall. We shall show that such variations cannot directly determine tip shape, but that they can determine the value of the so-called allometric coefficient.

\section{HYPOTHESIS}

We show first that Reinhardt's (1892) model - in which shape is determined by supply of material - is inadequate, and then develop D'Arcy Thompson's (1917) model in which wall elasticity determines shape.

We consider an extension zone of circular cross-section whose shape is otherwise arbitrary. We suppose that primary wall material is being supplied by flow through the tip with the lines of flow parallel to the axis but with the density of material flow, $f(r)$, possibly varying with the distance from the axis. We suppose further that the rate of growth of any small element of area in the wall of the extension zone is determined by the amount of material supplied to that element. The thickness of the wall, $\rho$, is assumed to be constant (Trinci \& Collinge, 1975).

Consider a narrow annular strip of wall whose radius is $r$ and whose width is $\delta m$ (see Fig. 1). The total area of the strip is $2 \pi r \delta m$, so its volume is $2 \pi \rho r \delta m$. The specific growth rate of the wall is [Trinci \& Saunders, 1977, equation (9)]

$$
C\left(1+\frac{1}{K}\right) \frac{\mathrm{d} r^{K}}{\mathrm{~d} m}
$$

where $K$ is the allometric coefficient and $C$ is a constant. In a short time interval $\delta t$ the total increase in wall volume is therefore

$$
2 \pi r C \rho\left(1+\frac{1}{K}\right) \frac{\mathrm{d} r^{K}}{\mathrm{~d} m} \delta m \delta t
$$

In the same time interval the volume of wall material supplied is

$$
2 \pi r f(r) \delta r \delta t
$$

Note that the increase in wall volume depends on $\delta m$, the increment in length along the tip, whereas the rate of supply to the wall depends on $\delta r$, the increment in the radius of the tip. Consequently the specific growth rate of the wall at a particular point on the extension zone depends not only on the variation in the flow of material at different radii but also on the angle between the wall of the tip and the axis of the tip or, equivalently, on $\delta m / \delta r$ (see Fig. 1). Hence from equation (3) 


$$
2 \pi r f(r) \delta r=2 \pi r \rho C\left(1+\frac{1}{K}\right) \frac{\mathrm{d} r^{K}}{\mathrm{~d} m} \delta m=2 \pi r \rho C\left(1+\frac{1}{K}\right) \frac{\mathrm{d} r^{K}}{\mathrm{~d} r} \delta r
$$

i.e.

$$
f(r)=C \rho\left(1+\frac{1}{K}\right) K r^{K-1}
$$

Thus the variation in the rate of supply of material is related to the allometric coefficient, $K$ ( $K$ is defined as the ratio of the longitudinal to the circumferential specific growth rate); in particular, if the flow is uniform so that $f(r)$ is a constant then $K$ must be unity and the growth is isotropic. The shape of the extension zone, which is represented in these equations by $\mathrm{d} m / \mathrm{d} r$, has, however, disappeared from the equations, and we conclude that the shape of the tip is not directly related to the variation in the rate of supply of material to the wall and, a fortiori, is not determined by it.

It is not difficult to see why this is so. Imagine an annular strip of wall moving back from the apex. As the cross-section of the tip increases in radius, the strip must expand transversely. If no new wall material is being supplied, then it can still accomplish this by shrinking in the longitudinal direction so as to maintain a constant area and thickness. The specific growth rates in the two directions will be equal in magnitude but opposite in sign, so the allometric coefficient will be - 1 . If, on the other hand, new wall material is being supplied, then, depending on the rate of supply, the strip can shrink less rapidly or even expand longitudinally. So the variation in rate of supply of material to the wall determines the allometric coefficient, not the shape of the extension zone.

In attempting to account for the shape of the hyphal tip by relating it to the supply of material to the wall, we have been implicitly assuming a particular model of tip growth. This model, originally due to Reinhardt (1892), treats the wall as a rigid layer, with the expansion of each small area of the surface due to the intussusception of a suitable amount of material in that area itself. As we have just seen, this model cannot account for the shape of the tip, and so we consider an alternative model, due to D'Arcy Thompson (1917). The wall is now considered to be elastic, and the shape is determined not by the pattern of addition of material but instead by the wall adjusting itself in response to turgor pressure so as to adopt a shape which minimizes the surface energy. The term 'elastic' is used to imply that the wall is flexible and can therefore adjust its shape to the dictates of turgor pressure.

Consider a surface separating two continuous media. Let $P$ be the difference in pressure across the surface and let $R_{\mathbf{1}}$ and $R_{\mathbf{2}}$ be the principal radii of curvature of the surface. [For our purposes it suffices to know that $R_{1}$ and $R_{2}$ measure, in a comparatively straightforward way, the degree to which the surface is curved; precise definitions and derivations of the formulae for the curvatures can be found in any text on classical differential geometry, such as Weatherburn (1927).] Then Laplace's formula (see Landau \& Lifshitz, 1959, p. 231) states that

$$
P=\alpha\left(R_{1}+R_{2}\right)
$$

where $\alpha$ is the surface tension coefficient. In our case we have an actual material surface, i.e. the primary wall of the tip, but the same formula applies providing we interpret $\alpha$ as expressing the resistance to stretching of the surface, however it arises.

If $P$ and $\alpha$ are both constant over the entire surface, then equation (6) implies that the surface must be spherical, as in the case of a soap bubble. If, however, the elasticity of the surface is not constant, then the shape may be quite different.

For tips whose cross-sections are circular the determination of the shape is in principle relatively straightforward. We take the axis of the tip as the $x$-axis and take co-ordinates $y$ and $z$ at right angles to the axis in the usual way. The equation of the surface of the tip can be written

$$
x=g(r)
$$




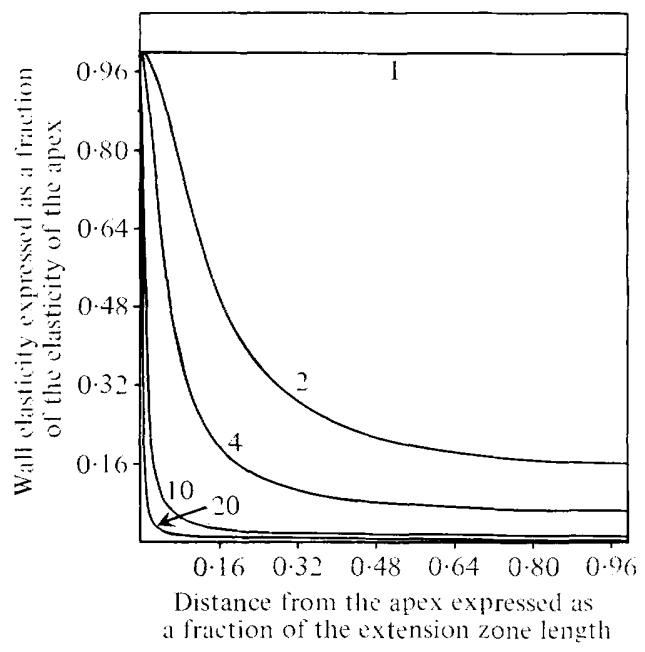

Fig. 2. Elasticity of the tip wall as a function of distance from the apex for different values of the ratio (length of extension zone)/(radius of tip). For ease of comparison, the distance is expressed as a fraction of the total distance from the apex to the end of the extension zone (measured along the surface of the tip) and the elasticity is expressed as a fraction of the elasticity at the apex.

where $r^{2}=y^{2}+z^{2}$. The sum of the principal curvatures is then given by (Weatherburn, 1927, p. 77)

$$
R_{1}+R_{2}=\frac{\mathrm{d}^{2} g}{\mathrm{~d} r^{2}}\left[1+\left(\frac{\mathrm{d} g}{\mathrm{~d} r}\right)^{2}\right]^{-\frac{3}{2}}+\frac{1}{r} \frac{\mathrm{d} g}{\mathrm{~d} r}\left[1+\left(\frac{\mathrm{d} g}{\mathrm{~d} r}\right)^{2}\right]^{-\frac{1}{2}}
$$

and on substituting this into equation (6) we obtain a differential equation for $g(r)$ which can be solved to give us the shape of the tip. Thus this model does lead to a definiteprediction.

The main difficulty that we anticipate in this procedure is that we are unlikely to know how elasticity varies along the tip. Accordingly it seems more profitable to use equation (6) the other way round, so to speak, by supplying observations about the shape of the tip and showing what these imply about the rigidity at different points. We have done this for the ellipsoidal shapes we discussed in an earlier paper (Trinci \& Saunders, 1977) and the results are illustrated in Fig. 2.

\section{DISCUSSION}

Ortega (1976) studied the stress behaviour of walls of stage IVb sporangiophores of Phycomyces blakesleeanus. Walls of growing sporangiophores could be represented by the Maxwell linear viscoelastic model which consists of a spring element and a viscous fluid element coupled in series; walls of dead sporangiophores behaved like a solid. During growth the sporangiophore wall could be modelled as a Newtonian viscous fluid and the rate of flow (i.e. extension rate) was a function of wall viscosity and the turgor pressure of the protoplast. Ortega suggested that the wall is a single regulatory parameter (as is also assumed here) and that wall viscosity is the most important parameter in regulating extension. Thus changes in wall viscosity may affect 'elasticity' (i.e. flexibility) of the extension zone wall.

It is not known what causes the elasticity of newly deposited primary wall material to decrease rapidly with time; it is certaintly not associated with any substantial increase in wall thickness (Trinci \& Collinge, 1975; Collinge et al., 1978).

Microfibrils (usually chitin or cellulose) form an important skeletal element of the walls of hyphae and may therefore be involved in determining its elastic properties. For example, wall elasticity may decrease as a consequence of an increase in microfibril thickness (Hunsley 
\& Burnett, 1968), an increase in the density of microfibril packing (Burnett, 1976), an increase in microfibril length (the elasticity of man-made composites is inversely related to polymer length) or the formation of cross-linkages between microfibrils. Park \& Robinson (1966) and Bartnicki-Garcia (1973) proposed that primary wall growth involves the action of enzymes which synthesize (e.g. chitin synthase) and hydrolyse (e.g. chitinase) wall polymers; the latter enzymes are called lysins. This hypothesis would explain wall intussusception in the model of tip growth proposed by Reinhardt (1892). Thus, according to the hypothesis of Bartnicki-Garcia wall elasticity might reflect the distribution of lysin activity in the tip wall. However, although lysins are certainly involved in branch initiation there is as yet no direct evidence that they are involved in tip growth.

Results of Katz \& Rosenberger with a temperature-sensitive mutant of Aspergillus nidulans suggest that microfibrils may not in fact be the crucial factor which determines tip shape; the mutant could not synthesize glucosamine and therefore chitin at the restrictive temperature (Katz \& Rosenberger, 1970). The walls of hyphae of the mutant grown at $41{ }^{\circ} \mathrm{C}$ contained only 7 to $15 \%$ of the normal chitin complement but maintained their normal shape when grown in medium containing an osmotic stabilizer or when transferred to buffer (Katz \& Rosenberger, 1971). Thus chitin microfibrils may not determine tip shape in this organism; certainly there can be a considerable reduction in chitin content without any effect on hyphal shape.

\section{REFERENCES}

BARTNiCKi-Garcia, S. (1973). Fundamental aspects of hyphal morphogenesis. Symposia of the Society for General Microbiology 23, 245-267.

BurnetT, J. H. (1976). Fundamentals of Mycology. London: Edward Arnold.

Collinge, A. J., Miles, E. A. \& Trinci, A. P. J. (1978). Ultrastructure of Penicillium chrysogenum hyphae from colonies and chemostat cultures. Transactions of the British Mycological Society 70, 401-408.

Hunsley, D. \& BurnetT, J. H. (1968). Dimensions of microfibrillar elements in fungal walls. Nature, London 218, 462-463.

Katz, D. \& Rosenberger, R. F. (1970). A mutation in Aspergillus nidulans producing hyphal walls which lack chitin. Biochimica et biophysica acta 208, 452-460.

Katz, D. \& Rosenberger, R. F. (1971). Lysis of an Aspergillus nidulans mutant blocked in chitin synthesis and its relation to wall assembly and wall metabolism. Archiv für Mikrobiologie 80, 284-292.

Landau, L. D. \& Lifshitz, E. M. (1959). Fluid Mechanics. Oxford: Pergamon.

ORTEGA, J. K. E. (1976). Mechanical and structural dynamics of cell wall growth. Ph.D. thesis, University of Colarado, Boulder, U.S.A.

PARK, D. \& Robinson, P. M. (1966). Aspects of hyphal morphogenesis in the fungi. In Trends in Plant Morphogenesis, pp. 27-44. Edited by E. G. Cutler. London: Longmans, Green \& Co.
ReinhardT, M. O. (1892). Das Wachstum der Pilzhyphen. Ein Beitrag zur Kenntniss des Flächenwachstums vegetabilischer Zellmembranen. Jahrbuch für wissenschaftliche Botanik 23, 479-566.

Robertson, N. F. (1958). Observations on the effect of water on the hyphal apices of Fusarium oxysporum. Annals of Botany 22, 159-173.

Robertson, N. F. (1965). The fungal hypha. Transactions of the British Mycological Society 48, 1-8.

Robertson, N. F. \& Rizvi, S. R. H. (1968). Some observations on the water relations of the hyphae of Neurospora crassa. Annals of Botany 32, 279291.

Steele, G. C. \& Trinci, A. P. J. (1975). The extension zone of mycelial hyphae. New Phytologist 25, 583-587.

Thompson, D'Arcy W. (1917). On Growth and Form. Cambridge: Cambridge University Press.

Trinci, A. P. J. (1978). Wall and hyphal growth. Science Progress, Oxford 65, 75-99.

Trinci, A. P. J. \& Collinge, A. J. (1975). Hyphal wall growth in Neurospora crassa and Geotrichum candidum. Journal of General Microbiology 91, 355-361.

TrincI, A. P. J. \& Saunders, P. T. (1977). Tip growth of fungal hyphae. Journal of General Microbiology 103, 243-248.

WeATHerburn, C. E. (1927). Differential Geometry of Three Dimensions. Cambridge: Cambridge University Press. 